\title{
A Novel Low Computational Complexity Multiple Signal Representation Technique for PAPR Reduction in OFDM Systems
}

\author{
Alok Joshi * and Nikita Airee \\ Department of ECE, Jaypee Institute of Information Technology, Noida 201 309, India; \\ nikita.jaypee@gmail.com \\ * Correspondence: 20.alok@gmail.com; Tel.: +91-120-4195885
}

\begin{abstract}
Orthogonal Frequency Division Multiplexing (OFDM) while being an efficient scheme for high data rate wireless communications has drawbacks such as higher Peak-to-Average Power Ratio (PAPR). To reduce PAPR, use of multiple signal representation technique such as Partial Transmit Sequence (PTS) is one of the favored techniques. However, the use of conventional PTS technique need excessive number of complex calculations in order to search for all permissible combinations of phase sequences causing steep increase in complexity in terms of complex computations. Paper aims to reduce the cumbersome process of phase selection by making use of the similarity of the phase vectors. The phase vectors are obtained sequentially and thus minimize the number of changes from one phase vector to another. Theoretical analysis shows that computational complexity is significantly reduced with the help of this proposed novel technique. We have also demonstrated that PAPR values are similar i.e. PAPR reduction capability remains similar but at reduced complexity.
\end{abstract}

Keywords: OFDM; PAPR; PTS

\section{Introduction}

The Orthogonal Frequency Division Multiplexing (OFDM) is a digital modulation method that splits a channel into larger set of narrow parallel sub-channels. Hence, it uses multiple-carriers for high rate data transmission. As the information is spread over the entire OFDM symbol duration, there is a greater tolerance to multipath fading and interference [1]-[2]. Moreover, efficient utilization of the spectrum makes OFDM a widely used technique. The bandwidth efficiency and reliability of the transmission system are further enhanced when MIMO-OFDM techniques are combined.

However, at the OFDM transmitter, high peak-to-average power ratio (PAPR) may be generated due to summation of subcarriers. When an OFDM signal with such high peaks is processed through a device with nonlinear characteristics e.g. a High Power Amplifier (HPA), due to harmonic generation signal distortion and takes place. Thus, HPA present at the OFDM transmitter needs to have a large input back off but that limits its efficiency. Methods [3] such as clipping [4], coding [5], probabilistic (scrambling) technique [6]-[11], pre-distortion and DFT-spreading among others have been adopted for the sake of reducing the PAPR in the OFDM systems. Probabilistic techniques are one of the most widely used of these methods. It can be implemented with the help of selected mapping (SLM) [8]-[10], partial transmit sequences (PTS), constellation extension, tone reservation-tone injection are the major techniques. In case of the conventional PTS (CPTS) scheme, input data is divided into several separate sub-blocks [11]. The Inverse Fast Fourier Transforms (IFFT) of all these sub blocks are then obtained and multiplied by phase rotation factors. Following this, they are optimally combined to form an OFDM signal with lower peaks or PAPR. However, with the use of the PTS scheme, there is an added complexity of searching for the optimal sequence that increases with the number of sub-blocks. Also, to ensure that correct the OFDM signal is 
recovered at the transmitter, additional information called side information is to be sent along with the data and is an overhead.

In this paper, we will deal with the issue of complexity in PTS. Hence, we need to devise a feasible method which would reduce the computations and hence decrease the complexity of the system making it more efficient. Several low complexity PTS techniques have been suggested [12]-[22]. Some of these like [13],[16] consider a preset threshold value of PAPR to decide a sequence for decreasing complexity. However, consideration of threshold leads to sub-optimal sequences. Moreover, most of these techniques give a significant reduction in multiplicative operations while the reduction in the number of computations for additive operations is not as significant. The proposed scheme gives significant reduction in both aspects of computational complexity utilizing the likeness of the candidate signals to sequentially obtain one from the other.

\section{Peak to Average Power Ratio and Multiple Signal Representation Techniques}

Being a multicarrier system OFDM is highly prone to large envelope variations leading to high peak to average power ratio. Peak to average power ratio is the ration peak power of the OFDM signal to the average power of the carrier. When PAPR is on the higher side it creates distortion when pass through a device with nonlinear I/O characteristic e.g. a high power amplifier. Multiple signal representation technique are one of the widely employed distortion less technique where data is divided in to sub blocks or scrambles and phase shifted using different combinations of phase vector, thus generating several OFDM symbols for same data set the one with the minimum power value will be used for transmission. Selective mapping and partial transmit sequence techniques are two such techniques. Partial transmit technique requires less number of complex arithmetic so used widely for many applications. Figure 1 shows various functional blocks of a PTS system.

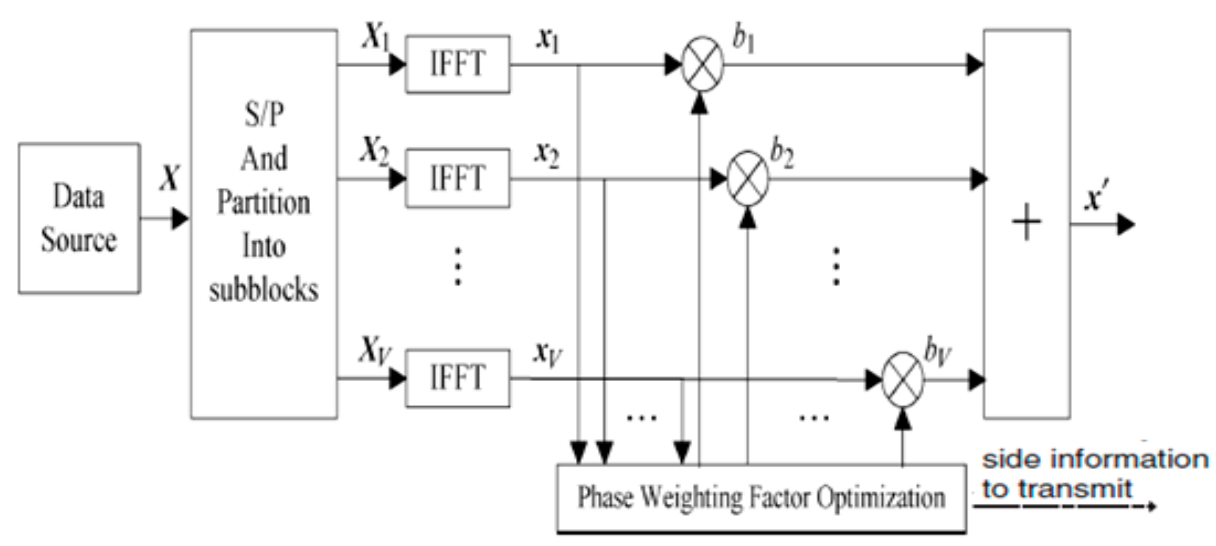

Figure 1. Typical functional blocks of PTS

The phase factor combination which rotates the existing constellation is selected in a manner that PAPR of transmitted OFDM signal $x^{\prime}$ is minimum.

$$
\left[\tilde{b}_{1}, \ldots \ldots \ldots . \widetilde{b}_{V}\right]=\underset{\left[b_{1} \ldots \ldots b_{V}\right]}{\arg \min }\left(\max _{n=0,1 \ldots . .1-1}\left|\sum_{v=1}^{V} b_{v} x_{v}[n]\right|\right)
$$

Where $V$ represents sub-blocks with $W$ phase weights, total numbers of permutations of phase weights which need to be analyzed are $W^{V-1}$, since for the first sub block rotating phase factor is always fixed as 1 . For $W$ phase weights, $W^{V-1}$ combinations are analyzed to get the candidate with least PAPR value. In generation of each sequence $V$-1 complex additions and same number of multiplication takes place. So total number of complex additions and multiplication can be given by: 
Complex additions $=(V-1) \cdot W^{V-1}$ and Complex multiplications $=(V-1) \cdot W^{V-1}$

E.g. for subblocks $(V=4)$ and $W=2\left(+1\right.$ and -1 or 0 representing $180^{\circ}$ shift $)$, the phase sequences would be $b_{1}, b_{2}, b_{3}, b_{4}$, since $b_{1}$ is always 1 , rest of the phase factors $b_{2}, b_{3}, b_{4}$ will have $8=2^{3}=W^{V-1}$ possible permutations, given in Table 1:

Table 1 Phase factor combinations for $W=2$ and $V=4$

\begin{tabular}{|l|c|c|c|c|c|c|c|c|}
\hline $\mathrm{b}_{2}$ & +1 & +1 & +1 & +1 & $-1 / 0$ & $-1 / 0$ & $-1 / 0$ & $-1 / 0$ \\
\hline $\mathrm{b}_{3}$ & +1 & +1 & $-1 / 0$ & $-1 / 0$ & +1 & +1 & $-1 / 0$ & $-1 / 0$ \\
\hline $\mathrm{b}_{4}$ & +1 & $-1 / 0$ & +1 & $-1 / 0$ & +1 & $-1 / 0$ & +1 & $-1 / 0$ \\
\hline
\end{tabular}

\section{Proposed Low Complexity PTS Scheme and Its Analysis with Results}

In this scheme we consider $W=2$ so that the possible values of phase weighting factors are represented by the set $\{1,-1\}$. It is assumed that the phase weight for the first sub-block $E_{1}$ is always constant at +1 . Therefore, it remains unchanged in all possible candidates. Considering sub-blocks sizes, $V=4$ for $W=2$, the following candidate signals are to be obtained:

$$
\begin{aligned}
& E_{1}+E_{2}+E_{3}+E_{4} \\
& E_{1}+E_{2}+E_{3}-E_{4} \\
& E_{1}+E_{2}-E_{3}+E_{4} \\
& E_{1}+E_{2}-E_{3}-E_{4} \\
& E_{1}-E_{2}+E_{3}+E_{4} \\
& E_{1}-E_{2}+E_{3}-E_{4} \\
& E_{1}-E_{2}-E_{3}+E_{4} \\
& E_{1}-E_{2}-E_{3}-E_{4}
\end{aligned}
$$

The number of computations required, according to the above equations, for varying values of $V$ is shown in Table 2.

Table 2: Number of computations required in Conventional PTS for W=2

\begin{tabular}{|c|c|c||}
\hline \multicolumn{3}{|c|}{ No. of computations for W=2 } \\
\hline \hline No. of sub-blocks & Additions & Multiplications \\
\hline \hline $\mathrm{V}=4$ & 24 & 24 \\
\hline $\mathrm{V}=5$ & 64 & 64 \\
\hline $\mathrm{V}=6$ & 160 & 160 \\
\hline $\mathrm{V}=7$ & 384 & 384 \\
\hline $\mathrm{V}=8$ & 896 & 896 \\
\hline
\end{tabular}

By simple observation, it can be inferred that the signals do not differ significantly. They can be arranged in such an order that they differ by only one phase factor:

$$
\begin{aligned}
& E_{1}+E_{2}+E_{3}+E_{4} \\
& E_{1}+E_{2}+E_{3}-E_{4} \\
& E_{1}+E_{2}-E_{3}-E_{4} \\
& E_{1}+E_{2}-E_{3}+E_{4}
\end{aligned}
$$




$$
\begin{aligned}
& E_{1}-E_{2}-E_{3}+E_{4} \\
& E_{1}-E_{2}-E_{3}-E_{4} \\
& E_{1}-E_{2}+E_{3}-E_{4} \\
& E_{1}-E_{2}+E_{3}+E_{4}
\end{aligned}
$$

By using simple mathematics, it can be determined that successive candidate signals arranged in the above order, differ only by certain fixed signals called difference signals:

$D_{2}=-2 E_{2}$

Number of Multiplications $=1$

$D_{3}=-2 E_{3}$

Number of Multiplications $=1$

$D_{4}=-2 E_{4}$

Number of Multiplications $=1$

In the proposed scheme, instead of computing each and every signal, we make use of these pre-calculated $D_{2}, D_{3}$ and $D_{4}$ to obtain the candidates signals by ensuring that there is only one change of phase factors between two successive candidate signals. To begin with, the first candidate signal is calculated such:

$$
S_{1}=A=E_{1}+E_{2}+E_{3}+E_{4}
$$

Number of Additions $=3$, Number of Multiplications $=3$

Now, subsequent signals are calculated, one from another, as they differ by only one term and the addition of a difference signal to the current candidate signal gives the next candidate signal.

$$
S_{2}=A+D_{4}=E_{1}+E_{2}+E_{3}-E_{4}
$$

Number of Additions $=1$, Number of Multiplications $=0$

$$
S_{3}=S_{2}+D_{3}=E_{1}+E_{2}-E_{3}-E_{4}
$$

Number of Additions $=1$, Number of Multiplications $=0$

$S_{4}=S_{3}-D_{4}=E_{1}+E_{2}-E_{3}+E_{4}$

Number of Additions $=1$, Number of Multiplications $=0$

$S_{5}=S_{4}+D_{2}=E_{1}-E_{2}-E_{3}+E_{4}$

Number of Additions = 1, Number of Multiplications $=0$

$S_{6}=S_{5}+D_{4}=E_{1}-E_{2}-E_{3}-E_{4}$

Number of Additions $=1$, Number of Multiplications $=0$

$S_{7}=S_{6}-D_{3}=E_{1}-E_{2}+E_{3}-E_{4}$

Number of Additions $=1$, Number of Multiplications $=0$

$S_{8}=S_{7}-D_{4}=E_{1}-E_{2}+E_{3}+E_{4}$

Number of Additions $=1$, Number of Multiplications $=0$

Therefore, total number of additions $=10$ while the total number of multiplications $=6$.

A measure of reduction of computational complexity is Computational Complexity Reduction Ratio (CCRR), is evaluated using following expression:

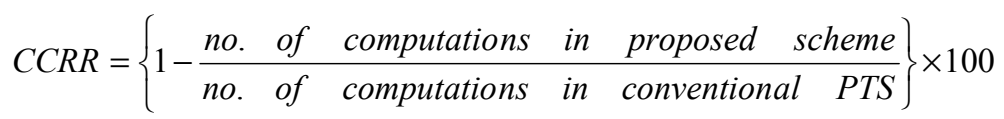

For $V=4$, the $C C R R$ calculated is:

$$
\begin{aligned}
& C C R R_{+}=\left\{1-\frac{10}{24}\right\} \times 100=58.33 \% \\
& C C R R_{\times}=\left\{1-\frac{6}{24}\right\} \times 100=75 \%
\end{aligned}
$$


here $C C R R_{+}$and $C C R R_{x}$ represent complexity reduction due to proposed scheme with respect to conventional PTS scheme in terms of addition and multiplication respectively.

Similarly, for $V=5$, we have the following difference signals:

$D_{2}=-2 E_{2}$

Number of Multiplications $=1$

$D_{3}=-2 E_{3}$

Number of Multiplications $=1$

$D_{4}=-2 E_{4}$

Number of Multiplications $=1$

$D_{5}=-2 E_{5}$

Number of Multiplications $=1$

Subsequently, the following candidate signals are obtained sequentially with the phase vectors following the simple rule that there is only one change of phase factors between two successive candidate signals

$S_{1}=A=E_{1}+E_{2}+E_{3}+E_{4}+E_{5}$

Number of Additions $=4$, Number of Multiplications $=4$

$S_{2}=A+D_{5}=E_{1}+E_{2}+E_{3}+E_{4}-E_{5}$

Number of Additions $=1$, Number of Multiplications $=0$

$S_{3}=S_{2}+D_{4}=E_{1}+E_{2}+E_{3}-E_{4}-E_{5}$

Number of Additions $=1$, Number of Multiplications $=0$

$S_{4}=S_{3}-D_{5}=E_{1}+E_{2}+E_{3}-E_{4}+E_{5}$

Number of Additions $=1$, Number of Multiplications $=0$

$S_{5}=S_{4}+D_{3}=E_{1}+E_{2}-E_{3}-E_{4}+E_{5}$

Number of Additions $=1$, Number of Multiplications $=0$

$S_{6}=S_{5}+D_{5}=E_{1}+E_{2}-E_{3}-E_{4}-E_{5}$

Number of Additions $=1$, Number of Multiplications $=0$

$S_{7}=S_{6}-D_{4}=E_{1}+E_{2}-E_{3}+E_{4}-E_{5}$

Number of Additions $=1$, Number of Multiplications $=0$

$S_{8}=S_{7}-D_{5}=E_{1}+E_{2}-E_{3}+E_{4}+E_{5}$

Number of Additions $=1$, Number of Multiplications $=0$

$S_{9}=S_{8}+D_{2}=E_{1}-E_{2}-E_{3}+E_{4}+E_{5}$

Number of Additions $=1$, Number of Multiplications $=0$

$S_{10}=S_{9}+D_{5}=E_{1}-E_{2}-E_{3}+E_{4}-E_{5}$

Number of Additions $=1$, Number of Multiplications $=0$

$S_{11}=S_{10}+D_{4}=E_{1}-E_{2}-E_{3}-E_{4}-E_{5}$

Number of Additions $=1$, Number of Multiplications $=0$

$S_{12}=S_{11}-D_{5}=E_{1}-E_{2}-E_{3}-E_{4}+E_{5}$

Number of Additions $=1$, Number of Multiplications $=0$

$S_{13}=S_{12}-D_{3}=E_{1}-E_{2}+E_{3}-E_{4}+E_{5}$

Number of Additions $=1$, Number of Multiplications $=0$

$S_{14}=S_{13}+D_{5}=E_{1}-E_{2}+E_{3}-E_{4}-E_{5}$

Number of Additions $=1$, Number of Multiplications $=0$

$S_{15}=S_{14}-D_{4}=E_{1}-E_{2}+E_{3}+E_{4}-E_{5}$

Number of Additions $=1$, Number of Multiplications $=0$

$S_{16}=S_{15}-D_{5}=E_{1}-E_{2}+E_{3}+E_{4}+E_{5}$ 
Number of Additions $=1$, Number of Multiplications $=0$

Here, total number of additions $=19$ and total number of multiplications $=8$.

Hence, for $V=5$ we have

$C C R R_{+}=\left\{1-\frac{19}{64}\right\} \times 100=70.31 \%$
$C C R R_{\times}=\left\{1-\frac{8}{64}\right\} \times 100=87.5 \%$

The same method is applied for $V=6$. So, we have the following difference signals and subsequent candidate signals, as obtained from the difference signals:

$D_{2}=-2 E_{2}$

Number of Multiplications $=1$

$D_{3}=-2 E_{3}$

Number of Multiplications $=1$

$D_{4}=-2 E_{4}$

Number of Multiplications $=1$

$D_{5}=-2 E_{5}$

Number of Multiplications $=1$

$D_{6}=-2 E_{6}$

Number of Multiplications $=1$

$S_{1}=A=E_{1}+E_{2}+E_{3}+E_{4}+E_{5}+E_{6}$

Number of Additions $=5$, Number of Multiplications $=5$

$S_{2}=S_{1}+D_{6}=E_{1}+E_{2}+E_{3}+E_{4}+E_{5}-E_{6}$

Number of Additions $=1$, Number of Multiplications $=0$

$S_{3}=S_{2}+D_{5}=E_{1}+E_{2}+E_{3}+E_{4}-E_{5}-E_{6}$

Number of Additions $=1$, Number of Multiplications $=0$

$S_{31}=S_{30}-D_{5}=E_{1}-E_{2}+E_{3}+E_{4}+E_{5}-E_{6}$

Number of Additions $=1$, Number of Multiplications $=0$

$S_{32}=S_{31}-D_{6}=E_{1}-E_{2}+E_{3}+E_{4}+E_{5}+E_{6}$

Number of Additions $=1$, Number of Multiplications $=0$

Here, total number of additions $=36$ and total number of multiplications $=10$.

Hence, for $V=6$ we have

$$
\begin{aligned}
& C C R R_{+}=\left\{1-\frac{36}{160}\right\} \times 100=77.5 \% \\
& C C R R_{\times}=\left\{1-\frac{10}{160}\right\} \times 100=93.75 \%
\end{aligned}
$$

These values can be calculated for any value of $V$ when $W=2$. It is inferred that the in proposed scheme the complex computations can be determined using following expressions:

No. of additions $=\left(W^{V-1}-1\right)+(V-1)=W^{V-1}+V-2$

No. of multiplications $=2 \times(V-1)$

Table 3 shows the values of CCRR for varying values of $V$ when $W=2$. 
Table 3: CCRR values for varying values of $\mathrm{V}$ when $\mathrm{W}=2$

\begin{tabular}{|c|c|c|}
\hline \multicolumn{3}{|c|}{ CCRR for W=2 } \\
\hline \hline Sub-blocks & CCRR + & CCRR $x$ \\
\hline $\mathrm{V}=4$ & $58.33 \%$ & $75 \%$ \\
\hline $\mathrm{V}=5$ & $70.31 \%$ & $87.5 \%$ \\
\hline $\mathrm{V}=6$ & $77.5 \%$ & $93.75 \%$ \\
\hline $\mathrm{V}=7$ & $82.03 \%$ & $96.87 \%$ \\
\hline $\mathrm{V}=8$ & $85.04 \%$ & $98.4 \%$ \\
\hline
\end{tabular}

The scheme proposed here reduces computational complexity as indicated by CCRR for complex additions as well as multiplications, with keeping the number of candidate signal unchanged. When CCRR is compared with what evaluated in [22] the CCRR $\times$ achieved is just $50 \%$, wherever in [23] CCRR $\times$ value of $85 \%$ is achieved for the sub-block size $V=8$ which is much less than $98.4 \%$ achieved in our scheme. Similarly in [24], [25] and [26] maximum reduction of $98 \%$ is achieved for the block size of $V=16$ which is bit difficult to implement than $V=8$ for similar values of CCRR. However in literature more emphasis is given on CCCRR $\times$, however in our paper the CCRR+ reduction is of similar order as in CCRR $\times$. Above all while implementing the proposed scheme for sake of the reducing computational complexity the PAPR reduction capability of PTS remains intact as shown in the Figure 2 as all the CCDF (complementary cumulative distribution function) are overlapping.

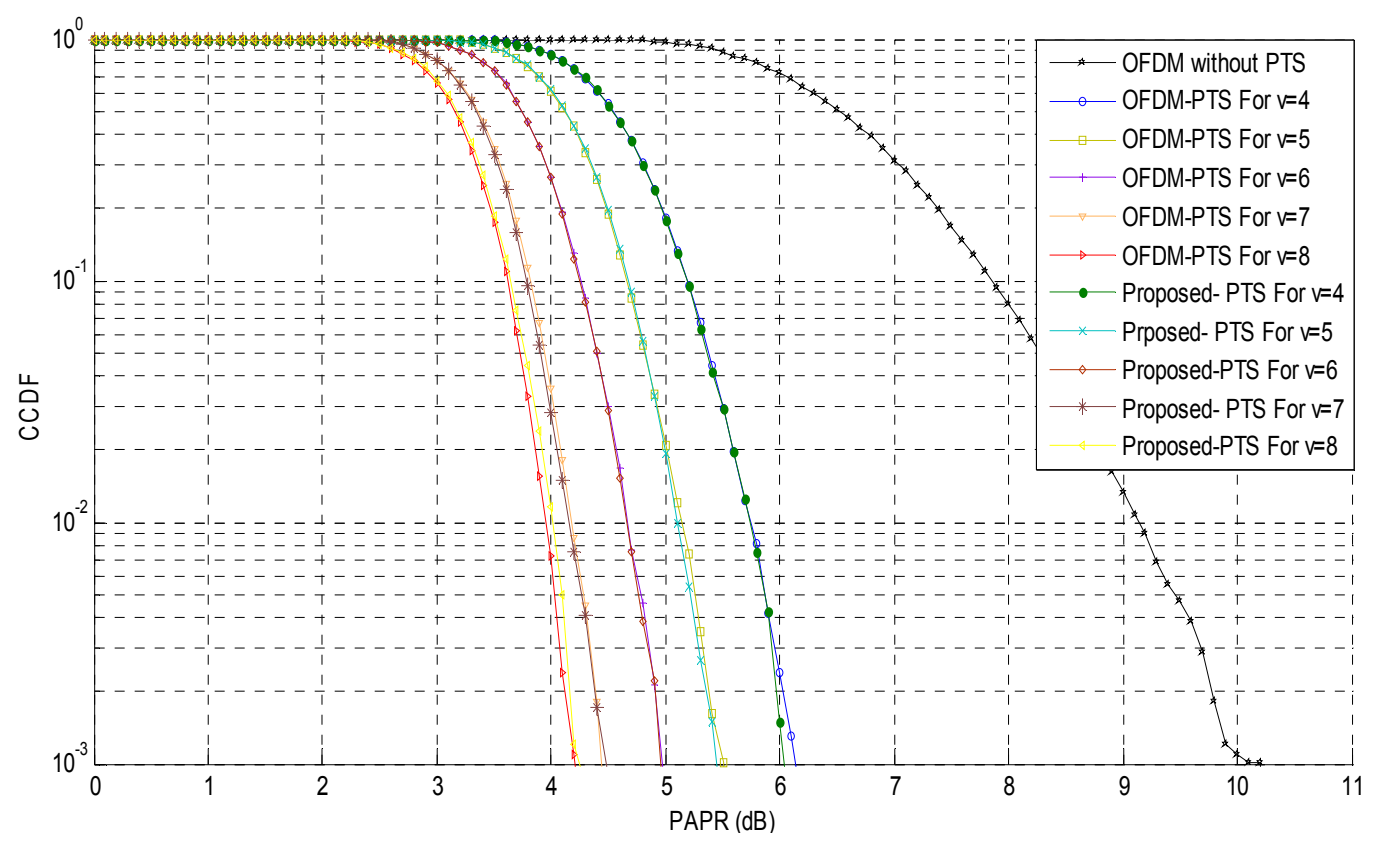

Figure 2. PAPR performance comparison of OFDM, OFDM-PTS and proposed PTS scheme.

\section{Conclusion}

Being most preferred method for high rate transmission OFDM suffers from high PAPR issues and PTS is the most effective solution for it. Proposed scheme offers encouraging reduction when 
computational complexity is concerned in PTS technique. The reduction in complex multiplication is as high as $98.4 \%$ and in complex additions almost $85 \%$ which would lead to an effective PTS with low complexity and PAPR reduction is similar to convention PTS scheme.

\section{References}

1. Author 1, A.B.; Author 2, C.D. Title of the article. Abbreviated Journal Name Year, Volume, page range, DOI or other identifier. Available online: URL (accessed on Day Month Year).

2. Yiyan $\mathrm{Wu} ; \mathrm{Zou}, \mathrm{W}$. Y. Orthogonal frequency division multiplexing: a multi-carrier modulation scheme. IEEE Transactions on Consumer Electronics 1995, vol. 41, no. 3, pp. 392-399.

3. Hwang, T. ; Yang, C. ; Wu, G. ; Li, S.; Li G. Y. OFDM and Its Wireless Applications: A Survey. IEEE Transactions on Vehicular Technology 2009, vol. 58, no. 4, pp. 1673-1694.

4. Han S. H.; Lee, J. H. An overview of peak-to-average power ratio reduction techniques for multicarrier transmission. IEEE Wireless Communications 2005, vol. 12, no. 2, pp. 56-65.

5. Gross, R. ; Veeneman, D. ; Clipping distortion in DMT ADSL systems. Electronics Letters 1993, vol. 29, no. 24, pp. 2080-2081.

6. Davis, J. A.; Jedwab, J. Peak-to-mean power control in OFDM, Golay complementary sequences, and Reed-Muller codes. IEEE Transactions on Information Theory 1999, vol. 45, no. 7, pp. 2397-2417, Nov 1999.

7. Kou, Y. J. ; Lu, W. S.; Antoniou, A. A New Peak-to-Average Power-Ratio Reduction Algorithm for OFDM Systems via Constellation Extension. IEEE Transactions on Wireless Communications 2007, vol. 6, no. 5, pp. 1823-1832, May 2007.

8. Wang, C. L. ; Tsai, Y. C. ; Ku, S. J. A Low-Complexity Constellation Extension Scheme for PAPR Reduction of OFDM Signals. Vehicular Technology Conference Fall (VTC 2009-Fall), 2009 ,pp. 1-5.

9. Bauml, R. W. ; Fischer, R. F. H. ; Huber, J. B. Reducing the peak-to-average power ratio of multicarrier modulation by selected mapping. Electronics Letters 1996, vol. 32, no. 22, pp. 2056-2057.

10. Breiling, H. ; Muller-Weinfurtner, S. H. ; Huber, J. B. SLM peak-power reduction without explicit side information. IEEE Communications Letter 2001, vol. 5, no. 6, pp. 239-241.

11. Wang, C-L.; Ouyang, Y. A low-complexity selected mapping scheme for peak-to-average power ratio reduction in OFDM systems. Vehicular Technology Conference, 2004 VTC2004-Fall. 2004 I, vol. 1, pp. 665-668..

12. Muller, S. H; Huber, J. B. OFDM with reduced peak-to-average power ratio by optimum combination of partial transmit sequences. Electronics Letters 1997, vol. 33, no. 5, pp. 368-369.

13. Xiao, Y. ; Lei, X. ; Wen, Q. ; Li, S. A Class of Low Complexity PTS Techniques for PAPR Reduction in OFDM Systems. IEEE Signal Processing Letters 2007, vol. 14, no. 10, pp. 680-683.

14. Yang, L. ; Chen, R. S.; Siu , Y. M.; Soo, K. K. PAPR reduction of an OFDM signal by use of PTS with low computational complexity. IEEE Transactions on Broadcasting 2006, vol. 52, no. 1, pp. 83-86.

15. Varahram, P. ; Al-Azzo, W. F. ;Ali, B. M. A low complexity partial transmit sequence scheme by use of dummy signals for PAPR reduction in OFDM systems. IEEE Transactions on Consumer Electronics 2010, vol. 56, no. 4, pp. 2416-2420.

16. Hou, J. ; Ge, J. ; Li, J. Peak-to-Average Power Ratio Reduction of OFDM Signals Using PTS Scheme With Low Computational Complexity. IEEE Transactions on Broadcasting 2011, vol. 57, no. 1, pp. 143-148.

17. Cimini, L. J. ; Sollenberger, N. R. Peak-to-average power ratio reduction of an OFDM signal using partial transmit sequences. IEEE Communications Letters 2000, vol. 4, no. 3, pp. 86-88.

18. Jiang, T. ; Xiang, W. ; Richardson, P. C. ; Guo, J. ; Zhu, G. PAPR Reduction of OFDM Signals Using Partial Transmit Sequences With Low Computational Complexity. IEEE Transactions on Broadcasting 2007, vol. 53, no. 3, pp. 719-724.

19. Wang, L.; Liu, J. PAPR Reduction of OFDM Signals by PTS with Grouping and Recursive Phase Weighting Methods. IEEE Transactions on Broadcasting 2011, vol. 57, no. 2, pp. 299-306.

20. Qi, X. ; Li, Y. ; Huang, H. A Low Complexity PTS Scheme Based on Tree for PAPR Reduction. IEEE Communications Letters 2012, vol. 16, no. 9, pp. 1486-1488.

21. Ghassemi, A.; Gulliver, T. A. A Low-Complexity PTS-Based Radix FFT Method for PAPR Reduction in OFDM Systems. IEEE Transactions on Signal Processing 2008, vol. 56, no. 3, pp. 1161-1166.

22. Lim, D. W. ; Heo, S. J. ; No, J. S.; Chung, H. A new PTS OFDM scheme with low complexity for PAPR reduction. IEEE Transactions on Broadcasting 2006 , vol. 52, no. 1, pp. 77-82. 
23. Han, S. H.; Lee, J. H. PAPR reduction of OFDM signals using a reduced complexity PTS technique. IEEE Signal Processing Letters 2004, vol. 11, no. 11, pp. 887-890.

24. Ye, C. ; Li, Z. ; Jiang, T.; Ni; C.; Qi, Q. PAPR Reduction of OQAM-OFDM Signals Using Segmental PTS Scheme With Low Complexity. IEEE Transactions on Broadcasting 2014, vol. 60, no. 1, pp. 141-147.

25. Cho, Y. J. ; No, J. S. ; Shin, D. J. A New Low-Complexity PTS Scheme Based on Successive Local Search Using Sequences. IEEE Communications Letters 2012, vol. 16, no. 9, pp. 1470-147.

26. Wang, Y. ; Chen, W. ; Tellambura,C. A PAPR Reduction Method Based on Artificial Bee Colony Algorithm for OFDM Signals. IEEE Transactions on Wireless Communications 2010, vol. 9, no. 10, pp. 2994-2999.

27. Taspinar, N.; Kalinli, A.; Yildirim, M. Partial Transmit Sequences for PAPR Reduction Using Parallel Tabu Search Algorithm in OFDM Systems. IEEE Communications Letters 2011, vol. 15, no. 9, pp. 974-976.

(C) 2016 by the authors; licensee Preprints, Basel, Switzerland. This article is an open access article distributed under the terms and conditions of the Creative Commons by Attribution (CC-BY) license (http://creativecommons.org/licenses/by/4.0/). 